\title{
Rapid and Coarse Face Detection in Cortex
}

\author{
Laura Cabral $^{\mathrm{a}}$, Bobby Stojanoski ${ }^{\mathrm{a}}$, and Rhodri Cusack ${ }^{\mathrm{a}, \mathrm{b}}$ \\ ${ }^{a}$ Brain and Mind Institute, Western University \\ ${ }^{b}$ Trinity College Institute of Neuroscience, Trinity College Dublin
}

\author{
Corresponding Author: \\ Laura Cabral \\ Brain and Mind Institute, \\ Western University, \\ London ON N6A 5B7 \\ Canada \\ lcabral@uwo.ca
}




\section{Abstract}

1 Humans have structures dedicated to the processing of faces, which include cortical

2 components (e.g. areas in occipital and temporal lobes) and subcortical components (e.g.

3 superior colliculus and amygdala). Although faces are processed more quickly than

4 stimuli from other categories, there is a lack of consensus regarding whether cortical or

5 subcortical structures are responsible for rapid face processing. In order to probe this, we

6 exploited the asymmetry in the strength of projections to subcortical structures between

7 the nasal and temporal hemiretina. Participants detected faces from unrecognizable

8 control stimuli and performed the same task for houses. In Experiments 1 and 3, at the

9 fastest reaction times, participants detected faces more accurately than houses. However,

10 there was no benefit of presenting to the subcortical pathway. In Experiment 2, we

11 probed the coarseness of the rapid pathway, making the foil stimuli more similar to faces

12 and houses. This eliminated the rapid detection advantage, suggesting that rapid face

13 processing is limited to coarse representations. In Experiment 4, we sought to determine

14 whether the natural difference between spatial frequencies of faces and houses were

15 driving the effects seen in Experiments 1 and 3. We spatially filtered the faces and houses

16 so that they were matched. Better rapid detection was again found for faces relative to

17 houses, but we found no benefit of preferentially presenting to the subcortical pathway.

18 Taken together, the results of our experiments suggest a cortical, coarse rapid detection

19 mechanism, which was not dependent on spatial frequency. 
Keywords: Rapid processing, face detection, subcortical, retinocollicular pathway, nasal temporal asymmetry. 


\section{Introduction}

22 Animals as diverse as fish, birds and sheep can recognize the faces of their

23 conspecifics (Leopold \& Rhodes, 2010). In humans there has evolved a network of

24 structures responsible for face processing that facilitate face detection, orientating, and

25 identification (Haxby, Hoffman, \& Gobbini, 2000; Mende-Siedlecki \& Verosky, 2013;

26 Tong, Nakayama, Moscovitch, Weinrib, \& Kanwisher, 2000). This comprises subcortical

27 components, including the superior colliculus and amygdala (Mende-Siedlecki \&

28 Verosky, 2013; Vuilleumier, Armony, Driver, \& Dolan, 2003), and cortical components

29 in the occipital and temporal lobes (Kanwisher, Mcdermott, \& Chun, 1997; Kanwisher \&

30 Yovel, 2006; Pitcher, Dilks, Saxe, Triantafyllou, \& Kanwisher, 2011). These specialized

31 processing mechanisms allow faces to be detected more quickly than objects (Crouzet,

32 Kirchner, \& Thorpe, 2010) and result in faces being the first category detected in visual

33 search tasks (Fletcher-Watson, Findlay, Leekam, \& Benson, 2008). Detecting faces

34 quickly is thought to be evolutionarily advantageous for both survival and social

35 interaction, from the savannahs of Africa to the office party.

36 The subcortical route via the retinocollicular pathway to the amygdala is often

37 thought to facilitate 'quick and dirty' face detection (Johnson, 2005). It comprises

38 projections from the retina to the superior colliculus, which in turn project to the pulvinar

39 nucleus on the way to the amygdala (Benevento \& Standage, 1983; Jones \& Burton,

40 1976; Rafal et al., 2015; Tamietto, Pullens, De Gelder, Weiskrantz, \& Goebel, 2012)

41 Evidence that the retinocollicular pathway can process faces comes from blindsight

42 patients, who after extensive damage to visual cortex are still able to detect the emotional

43 content of faces, although they cannot recognize their identity (Tietto \& de Gelder, 2010). 
44 Similar behavior is found in healthy controls following transracial magnetic stimulation

45 to the visual cortex; when TMS prevents participants from seeing stimuli, they are still

46 able to recognize the emotional content of the face (Jolij \& Lamme, 2005). Furthermore,

47 structures in the retinocollicular pathway are activated by the viewing of neutral and

48 emotional faces, as shown with functional magnetic resonance imaging (fMRI) (Mende-

49 Siedlecki \& Verosky, 2013). Functional magnetic resonance imaging has also found that

50 this pathway has a preference for crude, low-spatial frequency information, with greater

51 activation to faces filtered to emphasize low spatial frequencies than high spatial

52 frequencies (Vuilleumier et al., 2003).

53 Intracranial recordings in epilepsy patients have found that the retinocollicular

54 pathway is fast, with neural firing in the amygdala as quickly as $100-250 \mathrm{~ms}$ after the

55 presentation of an emotional face (Sato et al., 2013). Recent intracranial recording from

56 Méndez-Bértolo et al. (2016) has found even faster processing for fearful faces, with

57 firing in the amygdala recorded $74 \mathrm{~ms}$ after stimulus onset. Magnetoencephalography

58 (MEG) data suggests even faster processing with responses to emotional faces detected in

59 just 40 ms (Luo et al., 2010). Supporting this hypothesis, Garvert, Friston, Dolan, \&

60 Garrido (2014) used dynamic causal modeling of MEG data to conclude that a model

61 with a subcortical component, containing the pulvinar nucleus and the amygdala, more

62 accurately modeled rapid face processing than a model with a singular cortical process.

63 Fast detection mechanisms are not limited to subcortical structures, as there is also

64 evidence of rapid mechanisms within cortical areas, such as the inferior occipital gyrus

65 (Pitcher, Walsh, Yovel, \& Aviv, 2007; Sadeh, Podlipsky, Zhdanov, \& Yovel, 2010).

66 Specifically, an initial feed-forward wave of firing through cortex could allow for rapid, 
67 coarse processing (Cauchoix \& Crouzet, 2013; Serre, Oliva, \& Poggio, 2007; Vanrullen

$68 \&$ Koch, 2001). Electroencephalography (EEG) data from visual cortex can identify

69 responses just $56 \mathrm{~ms}$ after stimulus onset (Foxe \& Simpson, 2002), and intracranial

70 recordings in epilepsy patients found that the category of image participants were

71 viewing could be decoded from the first $100 \mathrm{~ms}$ of response in visual cortex (Liu, Agam,

72 Madsen, \& Kreiman, 2009). MEG data suggests occipitotemporal responses to faces in

73 just 100 ms (Liu, Harris, \& Kanwisher, 2002). Barragan-Jason, Cauchoix, \& Barbeau

74 (2015) have proposed that even the identification of familiar faces has an initial rapid

75 phase, occurring at $140 \mathrm{~ms}$, that depends on coarse visual information, and behavioural

76 responses to familiar faces can be detected in just $180 \mathrm{~ms}$ (Visconti di Oleggio Castello \&

77 Gobbini, 2015). To formalize how cortex could rapidly detect complex visual objects

78 such as faces in real-world scenes, Thorpe and colleagues (Delorme \& Thorpe, 2001;

79 VanRullen, Guyonneau, \& Thorpe, 2005) proposed a spike-based model of rapid

80 processing. These models have been supported by recordings from V1 in the macaque

81 and cat (Celebrini, Thorpe, Trotter, \& Imbert, 1993; Konig, Engel, Roelfsema, \& Singer,

82 1995; VanRullen et al., 2005).

83 In summary, there is evidence of both subcortical and cortical mechanisms for

84 rapid visual processing of faces. Which one, therefore, dominates rapid face detection in

85 healthy participants? One way to address whether rapid face perception is driven by

86 subcortical structures is to target the retinocollicular pathway to the amygdala. Presenting

87 stimuli exclusively to the nasal hemiretina preferentially targets the retinocollicular

88 pathway, as the nasal hemiretina contains more fibers projecting to the superior

89 colliculus. Initial evidence for this asymmetry came from tree shrews, cats and macaques 
90 (Conley, Lachica, \& Casagrande, 1985; Harrison, 2015; Perry \& Cowey, 1985; Pollack \&

91 Hickey, 1979; Sterling, 1973). fMRI evidence in humans has demonstrated that the

92 superior colliculus displays a temporal nasal asymmetry that is not found for the LGN or

93 V1 (Sylvester, Josephs, Driver, \& Rees, 2007). Additionally, behavioral studies have

94 demonstrated that a nasal-temporal asymmetry is reflective of input to the superior

95 colliculus. For example, making stimuli only visible to the $\mathrm{S}$ cones, which do not provide

96 input to the superior colliculus, eliminates the benefit of presenting to the nasal

97 hemiretina (Bertini, Leo, \& Làdavas, 2008).

98 Our goals in this study were to establish a paradigm for behaviorally quantifying

99 rapid face detection, and to determine whether presenting preferentially to the

100 retinocollicular pathway resulted in improved rapid face detection. Participants were

101 asked to detect faces from amongst unrecognizable control stimuli that were matched to

102 have the same low-level visual features, as quantified with a model of the early visual

103 system (Stojanoski \& Cusack, 2014). To determine whether any rapid detection

104 mechanism was specific to faces, we also tested a control condition requiring detection

105 another class of visual object, houses.

\section{2. Experiment 1}

\section{$107 \quad 2.1$ Methods}

108 To probe rapid face processing, in two blocks, participants performed a face detection

109 task in which they pressed a button as quickly as possible for intact faces, but not for

110 scrambled foil stimuli. In two additional blocks, they were asked to detect houses in a

111 similar manner. In each block, stimuli were presented monocularly, by asking

112 participants to wear an eye patch. This allowed us to target stimuli exclusively to either 
113 the nasal or temporal hemiretina. In the right eye, presenting stimuli to the right of

114 fixation targets the nasal hemiretina, while presenting to the left of fixation targets the

115 temporal hemiretina. The opposite is true in the left eye. Within each block, stimuli were

116 randomized across the nasal and the temporal hemiretinas.

117 2.1.1 Participants

118 Twenty-four individuals (12 males, 12 females, age range 18-21) were given course

119 credit for participation in Experiment 1. The non-medical ethics board at the University

120 of Western Ontario reviewed and approved the experimental protocol. All participants

121 gave informed consent, reported normal or corrected-to-normal vision, and that they were

122 right handed.

123 2.1.2 Stimuli

124 Twenty-four face photographs from an online database

125 (http://wiki.cnbc.cmu.edu/Face_Place) and 24 house stimuli, created by Martin, McLean,

126 O'Neil, \& Köhler (2013), were used in the study. As the house stimuli had a blurred

127 edge, a custom Matlab script added a blurred edge to the face stimuli, to appear similar

128 by eye. As the house stimuli were greyscale, face stimuli were also altered to be

129 greyscale.

130 All stimuli were centered in a rectangular area of 4.9 degrees by 4.9 degrees of visual

131 angle. The fixation cross was .5 degrees by .5 degrees. A white background was used

132 throughout the experiment. To generate the control stimuli, faces and houses were

133 diffeomorphically warped using the procedure described by Stojanoski \& Cusack (2014).

134 Foils were unrecognizable as determined by the behavioural ratings in Stojanoski \& 
135 Cusack (2014) (image 38 on the diffeomorphic continuum). A depiction of the stimuli 136 used in Experiment 1 can be found in Fig.1 A.

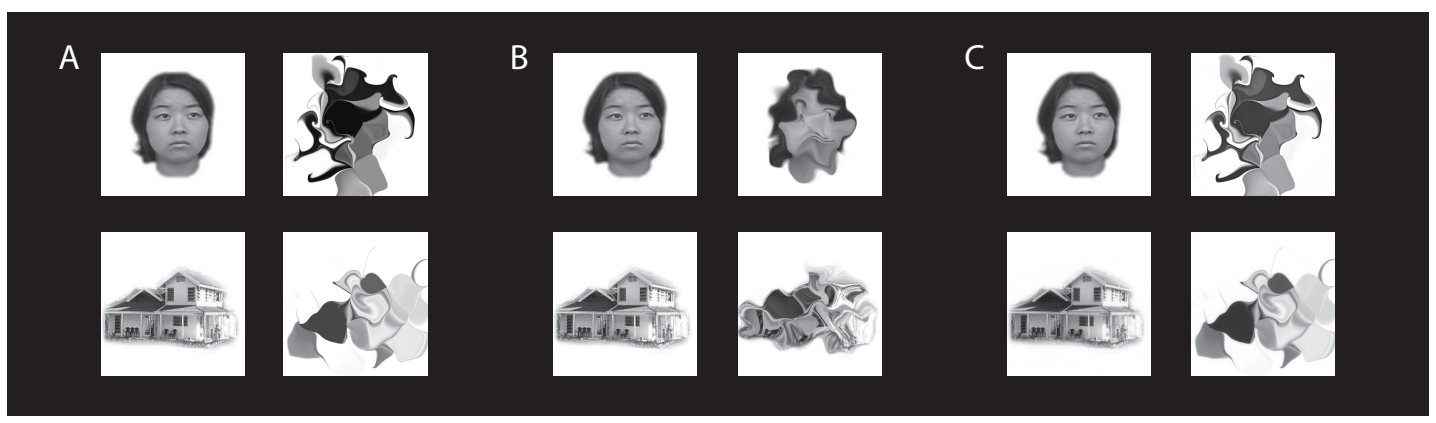

138 Figure 1. Exemplar stimuli from the four experiments. A) Stimuli used in Experiments 1

139 and 3. Foil stimuli are unrecognizable versions of faces and houses B) In Experiment 2,

140 the foil stimuli were more similar to the faces and houses. C) In Experiment 4, the stimuli

141 that were adjusted for differences in spatial frequency between categories.

142 2.1.3 Procedure

143 Stimuli were presented on a laptop screen using MATLAB and Psychtoolbox.

144 Participants wore an eye patch to ensure monocular presentation, placed their heads on a

145 chin rest, and were instructed to maintain fixation. The centre of the screen was directly

146 ahead of the nose. In each experimental block, a black fixation cross was offset by $3.2 \mathrm{~cm}$

147 to the left or right from center in order to put it directly in front of the unpatched eye.

148 This distance was chosen using the mean interpupillary distance scores from the 1988

149 Anthropometric Army Survey.

150 In experiment 1, participants completed two blocks with their left eye unpatched, one that

151 contained only face targets, the other containing house targets, and two similar blocks

152 with their right eye unpatched. Block order was counterbalanced across participants. 
153 In each block, participants were presented with 96 trials comprising two repetitions of 24

154 target stimuli and their 24 warped counterparts. One repetition was presented to the nasal

155 hemiretina, while the other was presented to the temporal hemiretina. To present to the

156 nasal and temporal visual hemiretina, the stimuli were offset horizontally so that the outer

157 edge of their rectangular bounds was 8 degrees from the center of fixation. Stimuli were

158 presented for duration of $122 \mathrm{~ms}$, with an inter-trial interval of $2505 \mathrm{~ms}$. Participants

159 were instructed to perform a simple detection task, pressing a key a quickly as possible

160 when they saw an intact face (in the face blocks) or an intact house (in the house blocks).

161 For a schematic of the experimental configuration, please see Fig. 2.

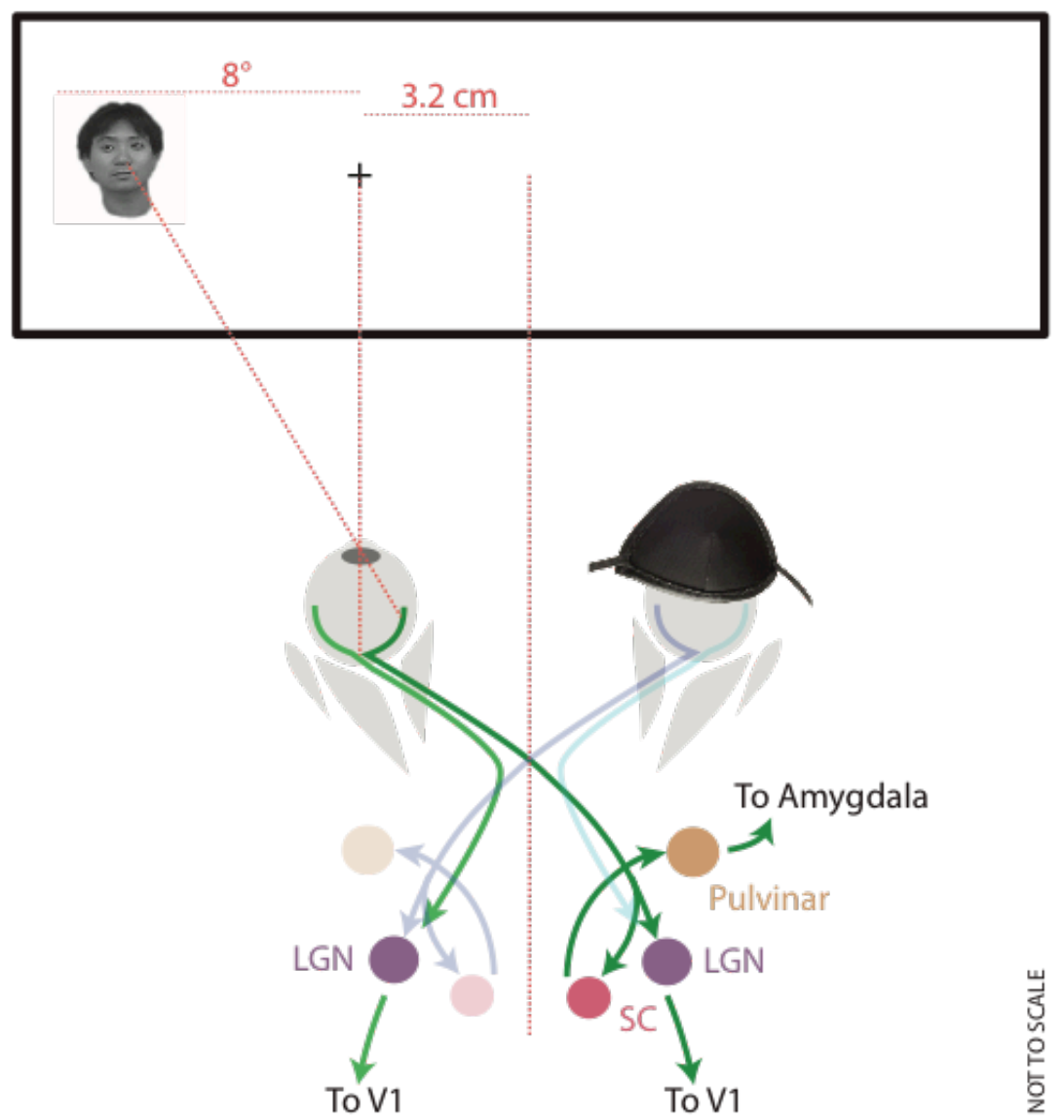

Figure 2. A schematic illustrating the experimental configuration. Participants wore an 164 eye patch to ensure monocular presentation and to allow the stimuli to be presented 
165 exclusively to the nasal or temporal hemiretina. The retinocollicular pathway is depicted

166 with projections from the nasal hemiretina to the superior colliculus, through the pulvinar 167 nucleus, terminating in the amygdala. Weaker, projections from the temporal hemiretina 168 to the superior colliculus are not shown.

169 2.1.4 Analysis

170 In order to quantify rapid processing, we used an analysis strategy similar to Kirchner \&

171 Thorpe's (2006) and calculated accuracy for the fastest $10 \%$ of responses. All reaction

172 times are relative to stimulus onset. A fast detection mechanism would be expected to

173 improve accuracy on these rapid trials by providing more accurate information to

174 decision and action areas sooner after stimulus onset. The reaction time (RT) threshold

175 for the fastest $10 \%$ of trials was calculated for each participant individually, in order to

176 account for individual differences in overall reaction time. We also expected that faces

177 would be detected more quickly overall. If this is the case, to ensure that the overall

178 difference in reaction time between the faces and houses did not drive the results, we

179 adopted a conservative analysis strategy and determined the face and house reaction

180 thresholds separately. Thus, the fastest $10 \%$ of face trials were expected to be even faster

181 than the fastest $10 \%$ of house trials.

182 To determine the contribution of the retinocollicular pathway, we examined whether

183 presenting the stimuli to the nasal or the temporal hemiretina modulated performance. As

184 the nasal hemiretina has more connections to the superior colliculus and thus the

185 retinocollicular pathway, we would expect to see faces more accurately detected than

186 houses, when the stimuli are presented to the nasal hemiretina. 


\section{$190 \quad 2.2$ Results}

191 Two participants were excluded for failing to follow the task instructions. Across

192 the remaining participants, mean reaction times for both the fastest $10 \%$ and slowest $50 \%$

193 of trials are shown in Fig. 3A. These reaction times include correct responses and false

194 alarms, as both contributed to subsequent accuracy metrics.
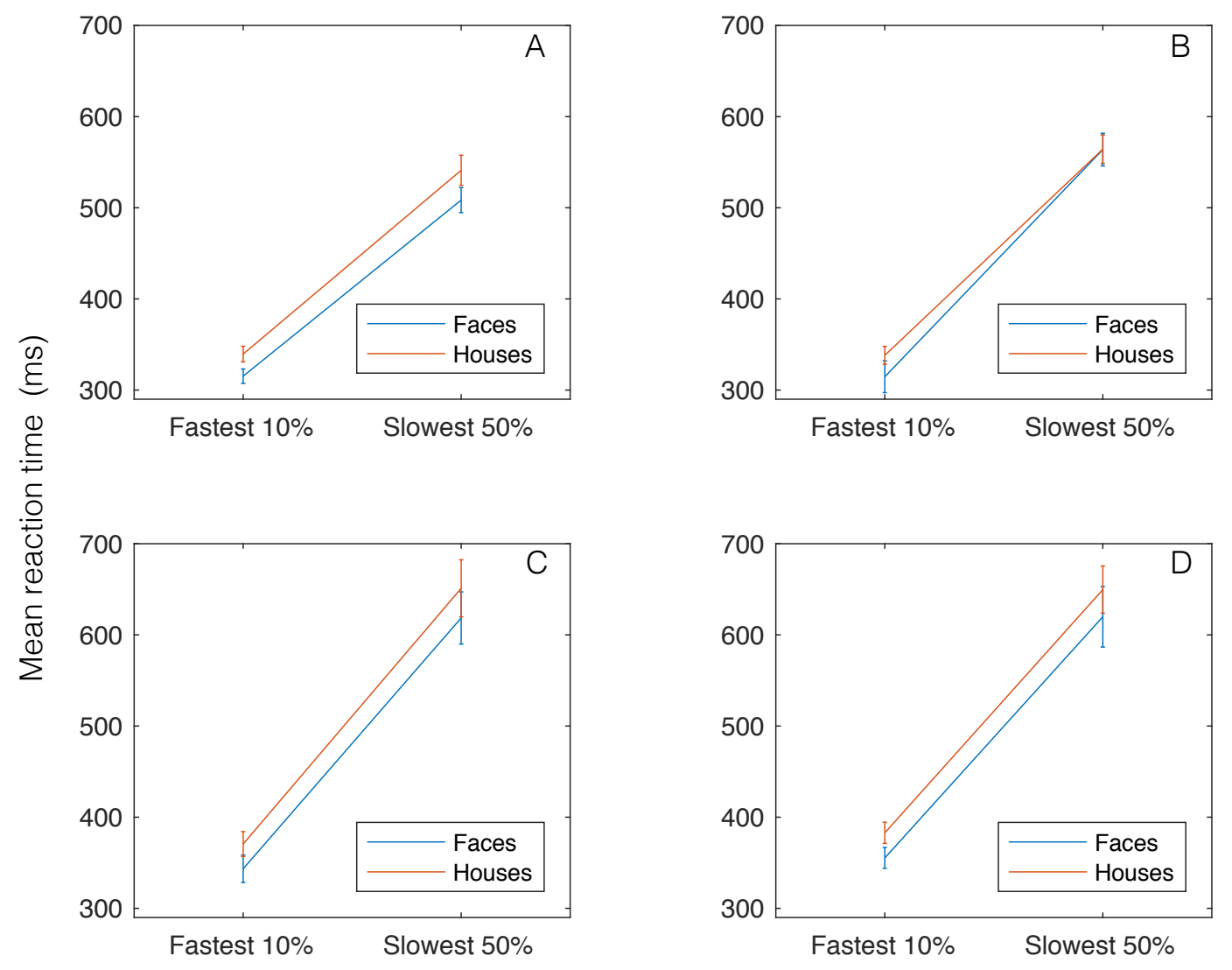

Trial subset

195

196 Figure 3. Mean reaction times for the fastest $10 \%$ and slowest $50 \%$ of trials A) In

197 Experiment 1, foil stimuli were unrecognizable versions of faces and houses B) In

198 Experiment 2, faces and houses were more similar to foil stimuli C) In Experiment 3,

199 stimuli were the same as in Experiment 1 D) In Experiment 4, spatial frequency of the

200 faces and houses were matched. Error bars represent plus-or-minus one standard error. 
202 To probe rapid mechanisms, analyses were confined to trials with a rapid response, in the 203 fastest $10 \%$ of RTs for each category. Participants were able to more accurately detect 204 faces than houses $(F(1,21)=10.41 p<0.01)$ (Fig. 4 A). This shows that our paradigm is 205 sensitive to rapid, accurate face detection. We then turned to the effect of the retinal 206 hemifield manipulation. There was no overall benefit of presenting stimuli to a particular 207 hemiretina $(F(1,21)=3.87, p>0.05)$, suggesting no general role for the retinocollicular 208 pathway in fast visual detection. Furthermore, contrary to what would be expected if the 209 retinocollicular pathway was category selective, and supported rapid face detection, there 210 was no significant stimulus by retinal hemifield interaction $(F(1,21)=0.1, p>0.05)$ (Fig.

$2115 \mathrm{~A})$. In fact, there was a trend for better performance for faces in the temporal hemiretina. 

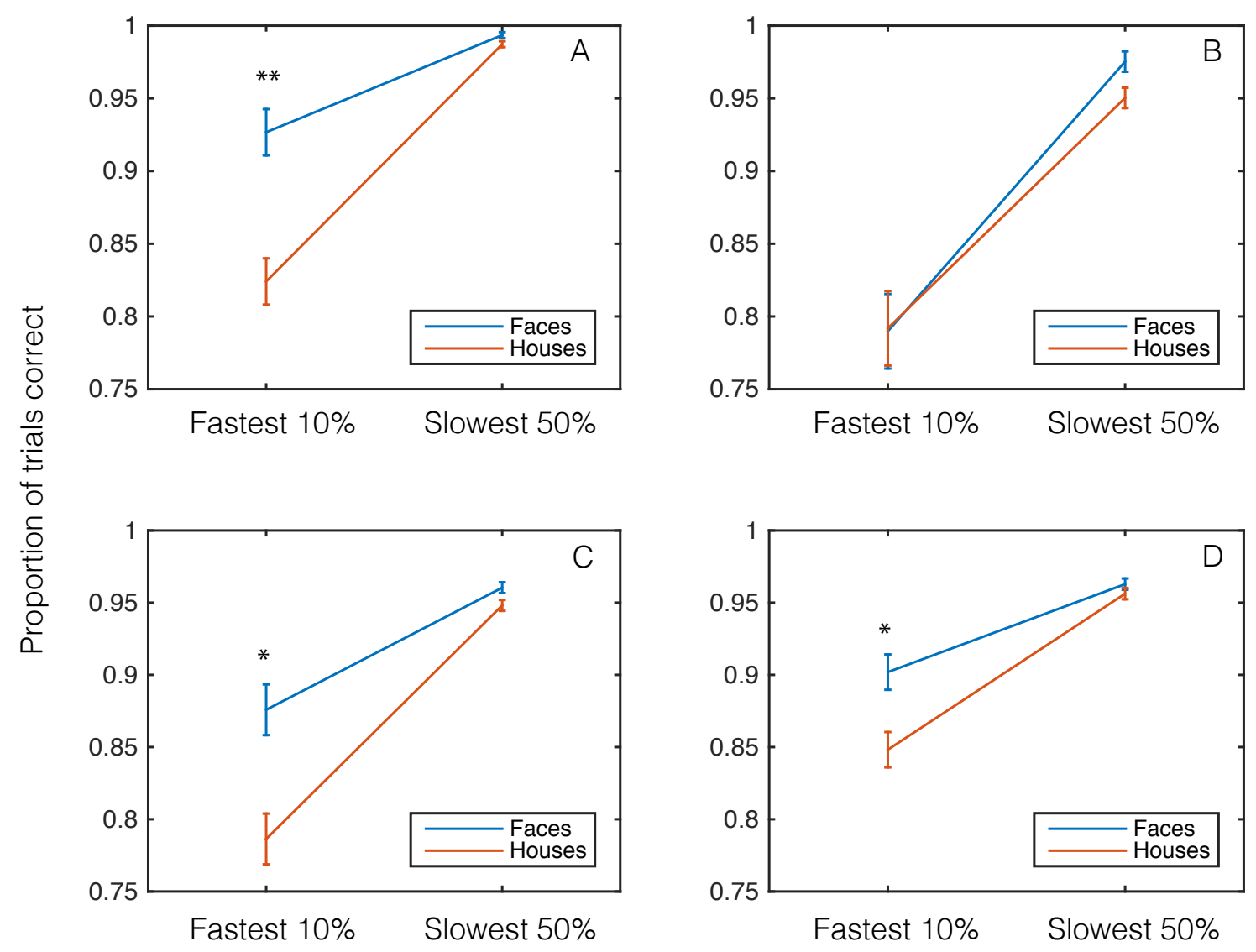

Reaction times

212

213 Figure 4. Proportion of trials correct, for the fastest $10 \%$ and slowest $50 \%$ of reaction

214 times. A) In Experiment 1, faces were detected significantly more accurately than houses

215 at the fastest reaction times. B) In Experiment 2, faces and houses were detected with

216 similar accuracy. C) Experiment 3 replicated the results of Experiment 1. D) In

217 Experiment 4, faces were detected significantly more accurately than houses in the fastest

$21810 \%$ of reaction times. In all experiments, error bars represent + - - the standard error. 


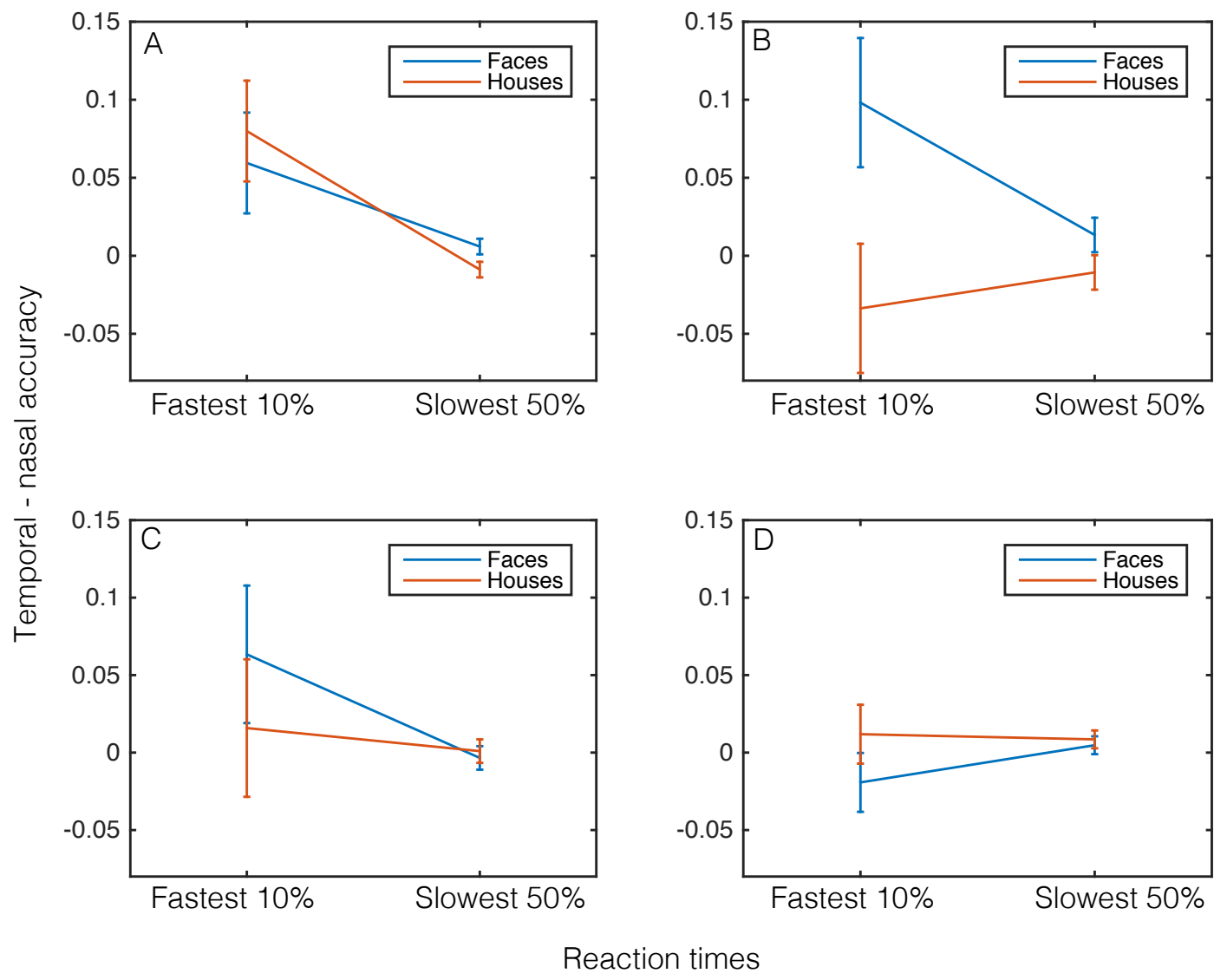

221 Figure 5. The difference in accuracy between the temporal and nasal hemiretina is plotted

222 for the fastest $10 \%$ and slowest $50 \%$ of RT's for Experiments 1-4 (A-D, respectively).

223 There were no significant differences between the nasal and temporal hemiretina for the

224 faces and houses in any of the experiments. Error bars represent plus-or-minus one

225 standard error.

\section{$226 \quad 2.3$ Interim Discussion}

227 The results of Experiment 1 demonstrate that there is a rapid route for detecting faces that

228 does not extend to other classes of stimuli (i.e. houses). As there was no benefit for

229 presenting stimuli to the nasal hemiretina, the results of the experiment did not provide 
230 any evidence of a role for the retinocollicular pathway in rapid visual detection or rapid

231 face processing. The lack of contribution from the retinocollicular pathway, taken with

232 the trend for better processing in the temporal hemiretina, suggests that a cortical route

233 could be responsible for the rapid face detection seen in the experiment.

234 Our next goal was to probe the specificity of a rapid cortical pathway. A key feature of

235 the rapid cortical route discussed in the literature is that it is not just quick, but that it is

236 dirty (i.e. a coarse representation). In an evolutionary context, it might be advantageous

237 for neural structures to obtain extremely quick, coarse representations of the faces in the

238 environment. This route is not thought to be capable of fine discrimination. Thus, the next

239 experiment was designed to probe the precision of the rapid detection mechanism

240 identified in Experiment 1.

\section{3. Experiment 2}

\section{$242 \quad 3.1$ Methods}

243 In order to examine the precision of the rapid cortical detection route, participants

244 performed the same task as in Experiment 1, but with less warped foil stimuli. These foil

245 stimuli still had some recognizable features of faces and houses. If detection relied on a

246 rapid route, exclusively for faces, it would support the idea that the rapid detection

247 mechanism was capable of precise representations. Otherwise, the rapid detection

248 mechanism might be limited to rapid, coarse judgments.

\section{3.1.1 Participants}

250 The same participants who participated in Experiment 1 participated in Experiment 2, and

251 the order in which participants completed the two experiments was counterbalanced.

252 Again, two participants were excluded for failing to follow the task instructions. 


\section{3}

254 The stimuli and procedure were the same as in Experiment 1, except that the foil images

255 had less warping applied (image 5 in the diffeomorphic continuum). Examples of the

256 stimuli can be found in Fig. 1B.

\section{$257 \quad 3.2$ Results}

258 As in Experiment 1, overall mean reaction times for the fastest $10 \%$ and slowest

$25950 \%$ of trials are shown in Fig. 3B. The mean reaction times include both correct

260 responses and false alarms. We used the same analysis procedure as in Experiment 1,

261 with accuracy in the fastest $10 \%$ of trials used to assess rapid face detection. When

262 participants were required to make precise judgments, faces were no longer detected

263 reliably more accurately than houses $(F(1,21)=0.08, N S)$ (Fig. 4B). Again, to examine the

264 role of the retinocollicular pathway we compared presentations to the nasal or the

265 temporal hemiretina. At the fastest RTs, there was no significant difference in accuracy

266 between hemiretinas $(F(1,21)=0.29, N S)$. Furthermore, again there was no evidence that

267 faces were detected significantly more accurately than houses in the nasal hemiretina

268 when compared to the temporal hemiretina $(F(1,21)=3.97$, NS (Fig. 5B).

269 In Experiment 1, we found evidence of a fast face processing mechanism when

270 faces were clearly distinct from foils. In Experiment 2, with a smaller difference between

271 faces and foils, we did not find the same effect. However, it is important to establish

272 whether the effect of the foil manipulation was significant, by directly testing whether the

273 results of the two experiments are significantly different. This comparison showed that

274 performance was significantly more accurate in Experiment 1 than in Experiment 2

$275(F(1,21)=6.81, p<0.05)$. Furthermore, there was a significant interaction between the 
276 experiments and stimulus type $(F(1,21)=8.03, p<0.05)$. This is driven by a greater

277 difference between rapid detection of faces and houses in Experiment 1 than in

278 Experiment 2.

\section{$279 \quad 3.3$ Interim Discussion}

280 When foil stimuli were created with less warping, requiring participants to make fine

281 discriminations, faces were no longer detected more accurately than houses at the fastest

282 reaction times. Again, there was not a significant advantage, or a trend for better

283 performance, when stimuli were preferentially presented to the retinocollicular pathway.

284 The results of this experiment support the idea that rapid detection of faces is limited to

285 coarse visual characteristics. When taking Experiment 1 and Experiment 2 together, the

286 results support the idea that there is a cortical mechanism capable of, and responsible for

287 rapid and coarse face processing.

288 One weakness of the current analysis that that the comparisons of the nasal and temporal

289 hemiretina contain half as much data as the collapsed analyses, and perhaps the

290 consequently reduced power that results is responsible for the lack of significance. Thus,

291 we conducted a further experiment, to double the number of subjects for this comparison.

292 Given recent concerns about the reproducibility of results in psychology (Open Science

293 Foundation, 2015) this also affords us the opportunity to test for replication of the other

294 findings from Experiment 1.

2954 Experiment 3

$296 \quad 4.1$ Methods

297 Experiment 3 was conducted to ensure that the results from Experiment 1 were

298 generalizable, replicating it in a different group of participants. In addition, we sought to 
299 combine the participants from Experiment 1 and Experiment 3 into a larger analysis,

300 where we would have increased power to detect differences in performance between the

301 nasal and temporal hemiretina.

302 4.1.1 Participants

303 Twenty-five self-reported right handed individuals (12 males, 13 females, age range 18-

304 42) participated in Experiments 3 and 4. Twenty-four participants reported normal or

305 corrected to normal vision. One participant did not have corrected to normal vision, their

306 prescription was +0.75 for the right eye and +0.5 for the left eye. Two participants were

307 excluded from the experiment, one because a fire alarm occurred during their

308 experimental session and the other because of technical difficulties that prevented button

309 presses from being recorded.

310 The participants received $\$ 10$ for their participation in the experiment. All participants

311 gave written informed consent. The non-medical ethics board at the University of

312 Western Ontario reviewed and approved the experimental protocol.

\section{4.1.2 Stimuli and Procedure}

314 Stimuli were identical to those that were used in Experiment 1. One important change

315 was made to the procedure. In order to gain information about the participants' reaction

316 times in both warped and intact trials, participants were instructed to press two buttons,

317 one for the warped images and another for the intact images. Exemplar images of the

318 stimuli can be found in Fig. 1A.

$319 \quad 4.2$ Results

320 Reaction times for the fastest $10 \%$ and slowest $50 \%$ of trials are shown in Fig. 3C. 
321 As in Experiments 1 and 2, accuracy in the fastest $10 \%$ of trials was examined. In this

322 experiment, we included the data from both the target and foil trials in our analysis.

323 Replicating the findings from Experiment 1, faces were detected significantly more

324 accurately than houses at faster RTs $(F(1,22)=6.24, p<0.05)$ (Fig. 4C). Again, when

325 collapsed across faces and houses, no difference in accuracy at fast RTs was found across

326 the nasal and temporal hemiretina $(F(1,22)=1.88 N S)$. Furthermore, the interaction

327 between the visual field and stimulus class showed that faces were not significantly more

328 accurate than houses in the nasal hemiretina than the temporal hemiretina $(F(1,22)=.19$,

329 NS) (Fig. 5C).

330 In order to test if a difference in response bias was responsible for the difference

331 in accuracy at the fastest reaction times, we also calculated the false alarm and hit rate for

332 the faces and houses. We were able to do this in Experiment 3 because it was a two-

333 button response task, which allowed us to bin all responses by RT. The mean false alarm

334 rate was lower for faces $(M=.21, S E=0.016)$ than for houses $(M=.25, S E=0.016)$ at the

335 fastest $10 \%$ of RT's. The mean hit rate was higher for faces $(M=.91, S E=0.017)$ than for

336 houses $(M=.82, S E=0.017)$ at the fastest $10 \%$ of RT's. A higher hit rate and a lower false

337 alarm rate shows the results were not driven by a response bias and were actually better at

338 identifying faces than houses. The higher hit rate and low false alarm rate for faces

339 suggests that participants were not merely responding less carefully to the rapid face trials

340 and that the results were not a result of a speed accuracy trade off.

341 Although a response bias does not appear to be causing the results in the

342 experiment, it is possible that the effect of hemifield is not being seen because of

343 insufficient power. Therefore, we conducted a further analyses in which we included 
344 participants from both Experiment 1 and Experiment 3 yielding $\mathrm{N}=45$. In both the nasal

345 and the temporal hemiretina, a significant difference in accuracy at fast reaction times

346 was found for face compared with house detection $(t(1,44)=2.03, p<0.05, t(1,44)=3.91$,

$347 p<0.001$, respectively). This supports the idea that increases in face detection accuracy

348 are not driven exclusively by an increase in performance in the nasal hemiretina, as

349 would be expected if the retinocollicular pathway were responsible.

$350 \quad$ Further combined analyses from Experiment 1 and Experiment 3 replicated the

351 key results. At the fastest reaction times, faces were detected more accurately than houses

$352(F(1,44)=16.44, p<0.001)$, consistent with the results of previous experiments. In

353 addition, at the fastest RTs, overall performance in the nasal hemiretina was significantly

354 worse than performance in the temporal hemiretina $(F(1,44)=5.74, p<0.05)$. With the

355 larger sample, there was still no significant interaction between stimulus and field

$356(F(1,44)=0.03, N S)$ as would be expected if a nasal benefit was driving improved face

357 detection.

\section{$358 \quad 4.3$ Interim Discussion}

359 Experiment 3 replicated the results of Experiment 1, generalizing the findings to a

360 different group of participants and a slightly different response procedure. In addition,

361 calculating the false alarm and hit rates allowed us to determine that a response bias was

362 not the cause of our results. A higher hit rate and a lower false alarm rate for faces

363 suggests that increased accuracy is not a result of a speed accuracy trade off. This is

364 further emphasised because faces have a faster mean reaction time than houses.

365 Combining the results from Experiments 1 and 3 into a single analysis revealed

366 that presentation to the nasal hemiretina led to significantly worse rapid detection of faces 
367 and houses. This result is contrary to what would be expected if the nasal hemiretina, and 368 thus the retinocollicular pathway, were driving the results. In addition, both the nasal and 369 the temporal hemiretina show evidence of significantly more accurate face detection at 370 fast reaction times, demonstrating that there is not one hemiretina driving the fast face 371 detection advantage.

372 Taken together, these results provide more support for the idea that a cortical, 373 rather than a subcortical, process is responsible for rapid face detection. Why might 374 presenting to the nasal hemiretina result in reduced detection of visual stimuli? It is 375 possible that reduced performance could be caused by distracting information (i.e. 376 emotional content) being communicated from subcortical structures to cortical structures.

377 At fast RTs, the brain might be only capable of attending only to a subset of information, 378 and emotional content might take precedence over visual categorization, decreasing the 379 accuracy of the nasal hemiretina in Experiments 1 and 3.

380 In a final experiment we control for a potential low-level visual explanation for the 381 category specificity of the rapid detection mechanism. In our stimulus sets (and more

382 generally, Awasthi, Sowman, Friedman, \& Williams, 2013), faces contained lower spatial 383 frequencies than houses. Natural images generally have greater power at lower

384 frequencies (Burton \& Moorhead, 1987) so perhaps we have more rapid mechanisms for 385 low spatial frequencies, that process faces more rapidly. Thus, perhaps spatial frequency, 386 rather than category per se is responsible for the category specific rapid detection we 387 observed in Experiments 1 and 3.

\section{Experiment 4}

$389 \quad 6.1$ Methods 
390 In Experiment 4 we repeated Experiment 3, but the face and house stimulus sets filtered

391 so that they had balanced power spectra.

392 6.1.2 Participants

393 Experiment 4 tested the same participants as Experiment 3, and the order in which they

394 participated was counterbalanced. Again two participants were excluded - one because of

395 a fire drill and the other because of technical difficulties that prevented button presses

396 from being recorded.

397 6.1.2 Stimuli and Procedure

398 The same stimuli that were used in Experiment 1 and 3 were used in Experiment 4, but

399 with the spatial frequency of the images balanced. Each image was transformed into 2D

400 frequency space using a Fourier transform. Each pixel was then multiplied by a scalar

401 filtering function that depended only on distance from the origin of frequency space.

402 Finally, an inverse Fourier transform was used to return to image space. Houses were

403 filtered to remove high spatial frequency information, and faces were filtered to remove

404 low spatial frequency information. A further processing stage was applied, to remove a

405 visually salient artefact, which was the bleeding of images into the background

406 surrounding them. All voxels outside of each object in the original image (i.e., that were

407 exactly background color) were reset to the background color after filtering. This led to a

408 slight residual mismatch in the resulting frequency spectra, which can be seen in the

409 original and final frequency spectra, shown in Fig. 6. Exemplar images can be found in

410 Fig, 1C. All other aspects of the experiment were the same as in Experiment 3. 


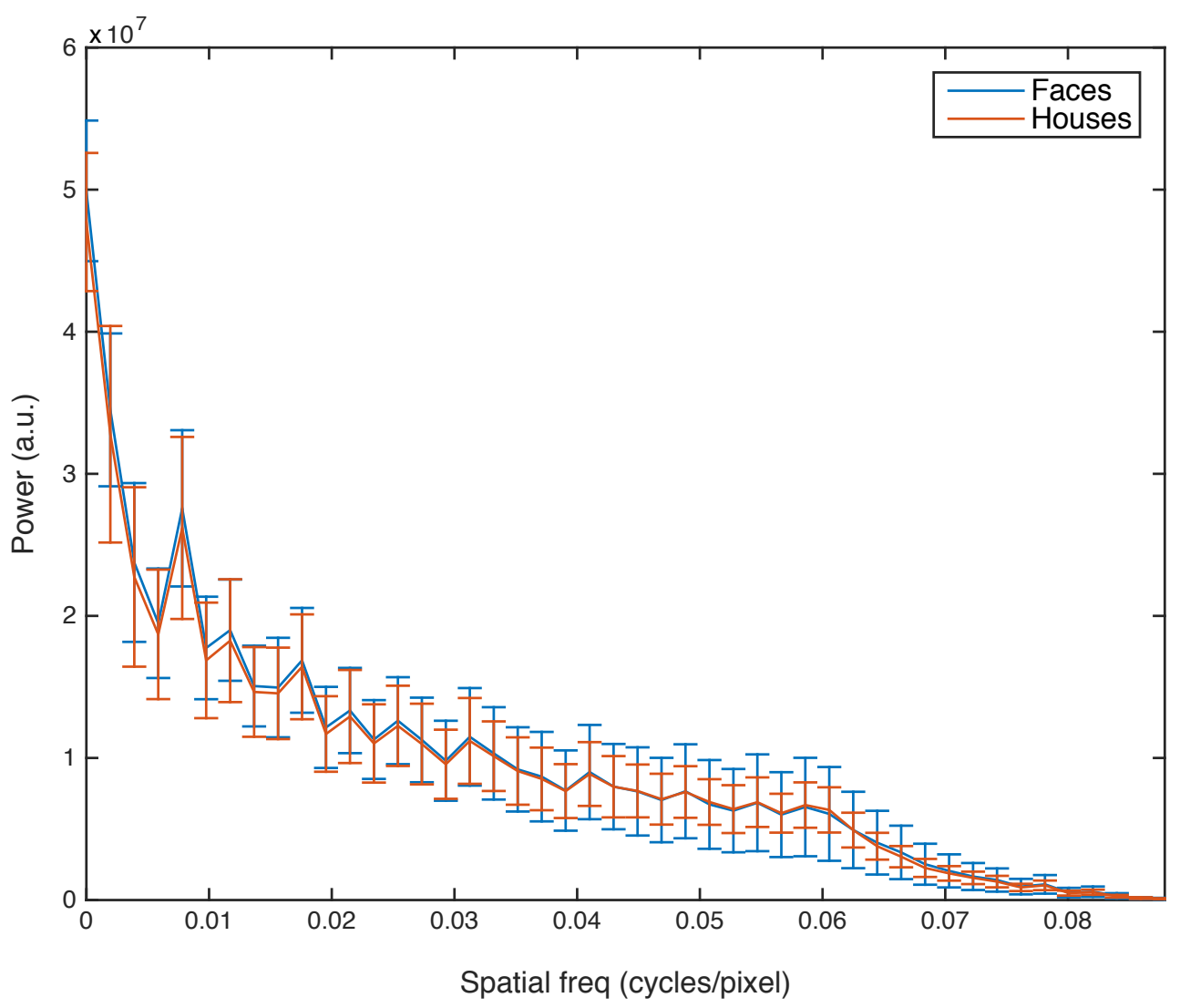

411

412 Figure 6. Power of the faces and houses at each spatial frequency (cycles/pixel).

\section{$413 \quad 6.2$ Results}

414 Reaction times for the fastest $10 \%$ of trials and the slowest $50 \%$ of trials can be found in 415 Fig. 3D.

416 As we obtained data from both target and foil trials, both were included in our analysis.

417 In the fastest $10 \%$ of trials, faces were again detected more accurately than houses,

418 despite the matching of spatial frequencies $(F(1,22)=4.83, p<0.05)$ (Fig. 4D).

419 Again, the contribution of the retinocollicular pathway was assessed. No significant

420 differences in accuracy were seen for the nasal compared with the temporal hemiretina $421(F(1,22)=0.02, N S)$. The interaction between hemiretina presentation and stimulus was 
422 also not significant; houses were not detected significantly more accurately than faces at

423 fast reaction times when contrasting the temporal with the nasal hemiretina $(F(1,22)=.67$,

$424 \quad N S)$ (Fig. 5D).

425 To investigate whether spatial frequency manipulation substantially modulated

426 performance, the fastest $10 \%$ of trials from experiment 3 were compared to the fastest

$427 \quad 10 \%$ of trials from experiment 4 using a $2 \times 2$ ANOVA with experiment and stimulus as

428 the within-subject factors. Overall, there were no significant difference in performance

429 between the two experiments $(F(1,22)=2.80, p>0.05)$. Furthermore, there was no

430 significant interaction between stimulus and experiment, showing the difference in

431 accuracy for faces compared with houses was not significantly different in experiments 3

432 and $4(F(1,22)=0.60, p>0.05)$. In line with the results of each experiment, there was a

433 main effect for stimuli, with faces detected significantly more accurately than houses at

434 the fastest reaction times $(F(1,22)=10.17, p<0.01)$.

435 To further investigate if response bias caused the differences in accuracy at the fastest

436 reaction times, we calculated the false alarm and hit rate for the faces and houses. The

437 mean false alarm rate was lower for faces $(M=.13, S E=0.018)$ than for houses $(M=0.15$,

$438 S E=0.018)$ at the fastest $10 \%$ of RT's. The mean hit rate was higher for faces $(M=.91$,

$439 S E=0.017)$ than for houses $(M=.83, S E=0.017)$ at the fastest $10 \%$ of RT's. A higher hit

440 rate and a lower false alarm rate again confirms that response bias cannot account for the

441 differences in accuracy, and that participants were better at identifying faces than houses.

\section{$442 \quad 6.3$ Interim Discussion}

443 This experiment explored the idea that spatial frequency might have caused the category-

444 specific effects in experiment 1 and 3. Altering the spatial frequency of the images did 
445 not have any significant effect on the results. In trials with fast RTs, faces were still

446 detected more accurately than houses. Furthermore, the spatial filtering in experiment 4

447 did not change the results from those seen in experiment 3 . These results support a face-

448 specific rapid detection mechanism, rather than a low-spatial frequency mechanism.

\section{General Discussion}

450 Four experiments were conducted to determine whether a rapid route for face detection

451 could be identified in a behavioural experiment. In addition we sought to determine

452 whether a cortical or a subcortical process, facilitated by the retinocollicular pathway,

453 could be responsible for the rapid detection of faces. If the retinocollicular pathway to the

454 amygdala were responsible for rapid face detection, we would expect to see a benefit for

455 faces, but not houses, when presenting to the nasal hemiretina. In Experiments 1, 3 and 4,

456 participants rapidly detected faces but not houses from very distinct warped foil stimuli.

457 However, there was no benefit of presenting the stimuli to the nasal hemiretina, providing

458 no support for a retinocollicular route in rapid face detection in our task. Even when we

459 combined the participants from Experiments 1 and 3 into a single analysis to increase

460 power, we did not see a benefit for face detection in the nasal hemiretina, and in fact,

461 faces or houses presented to the nasal hemiretina were detected less accurately.

462 We then considered what aspects of the face stimuli could have led to rapid

463 detection. Faces have greater power at lower spatial frequencies than houses. In

464 Experiment 4, we filtered the images to enhance relative power at high spatial

465 frequencies for the faces and reduce it for the houses. Faces were still detected more

466 accurately than houses, showing that it is category, and not just spatial frequency, that

467 facilitates rapid detection. Furthermore, we found performance overall was no worse 
468 when high frequencies were emphasized. This suggests low spatial frequencies did not

469 have a strong role, and that perhaps the rapid detection mechanism is capable of precise

470 visual representation. We tested this in Experiment 2 and found that when participants

471 were required to make fine visual discriminations, more accurate fast face detection

472 disappeared. This suggests the rapid discrimination method is "dirty" as well as being

473 "quick". Again no contribution was evident from the retinocollicular pathway.

474 Taken together, our results show there is a rapid route for the detection of faces,

475 which relies on coarse visual information, but not low on spatial frequencies in particular.

476 In none of the experiments did we find evidence of a benefit for face detection in the

477 nasal hemiretina. This supports the idea that a cortical rather than a subcortical

478 mechanism is responsible for rapid face detection (Cauchoix, \& Crouzet, 2013), and is

479 congruent with evidence that cortex is capable of rapid processing (Barragan-jason et al.,

480 2015; Foxe \& Simpson, 2002; H. Liu et al., 2009).

481 Strengthening the results of the study, the warped foil stimuli used in this

482 experiment were well matched in terms of luminance, contrast, and spatial frequency to

483 the target stimuli, and could not be differentiated in a model of the early visual system

484 (HMAX, Stojanoski \& Cusack, 2014), eliminating a series of confounding variables not

485 often considered. There is one study where the authors found participants were orienting

486 more quickly to 'face like' stimuli when they were presented to the nasal hemiretina

487 (Tomalski, Johnson, \& Csibra, 2009). However, this study used 'Johnson faces' where

488 black boxes are put in place of the eyes, nose and mouth. Control stimuli in this study

489 were an inverted version of the 'Johnson face'. Although these control stimuli were

490 matched for variables like spatial frequency, the target stimuli will have a large 'top- 
491 heavy' bias in comparison with the foil stimuli, which could be what was responsible for

492 the increased performance of the nasal hemiretina. Our naturalistic stimuli will likely

493 have had less of a top-heavy bias, and this could be a potential reason why we do not see

494 a benefit of presenting to the nasal hemiretina. It is also possible that cortex is needed to

495 make category judgements when target and foil stimuli are well matched.

496 In order to ensure that the visual stimuli were unrecognizable, Stojanoski and

497 Cusack (2014) quantified how much warping was necessary to remove semantic

498 information from different categories. Faces, along with bikes, needed the highest levels

499 of warping in order to render them unrecognizable. Therefore, it is unlikely that face

500 blocks in experiments 1 and 3 represented an easier task than house blocks. In addition,

501 different diffeomorphic fields were used for each foil, which makes them distinct, even

502 within a category. For example, although the first exemplar in Figure 1 has a dark portion

503 in the center, not all face stimuli have this. Across the entire face and house categories,

504 these small characteristics were insufficient to have driven the broader differences

505 between the faces and the houses.

506 Other researchers have also failed to see a benefit for face identification when

507 presenting stimuli to the nasal hemiretina (Gabay, Burlingham, \& Behrmann, 2014). In

508 addition to the nasal/temporal manipulation, these researchers use a Wheatstone

509 stereoscope to exploit the fact that visual information is segregated monocularly until

510 visual cortex. Gabay et al. (2014) present stimuli monocularly, either to the same or

511 different eyes and have participants make identity judgements. They find a benefit for

512 presenting stimuli to the same eye, which they hypothesize, could be due to the

513 monocular properties of subcortical structures, such as the lateral geniculate nucleus 
514 (LGN). Although the retinocollicular pathway does not seem to be contributing to

515 improved face detection, it is possible that the LGN, on the way to cortex, could be 516 partially responsible for our results.

517 If the amygdala is not responsible to the rapid detection of faces, it could still be 518 processing emotional information (Vuilleumier, Richardson, Armony, Driver, \& Dolan, 519 2004). This could explain why we see decreased overall performance for the nasal

520 hemiretina. It is possible that when the amygdala feeds information to the cortex creating

521 competing processing, which makes it more difficult for cortex to rapidly categorize

522 visual stimuli.

523 If faces are being detected more accurately at fast reaction times than the houses,

524 what features of the stimuli are causing this increase in accuracy? Faces have

525 significantly less inter-exemplar variability than houses. It is possible that the invariance

526 of face stimuli allows tighter tuning in cortex, leading to more accurate, robust and

527 efficient detection. If the invariability in our stimuli is causing the effects seen in the

528 experiments, it is possible that other stimulus categories with limited variability could tap

529 into a rapid mechanism. If other categories of stimuli could be capable of tapping into the

530 rapid mechanism, are faces really special or is expertise what is important in order to

531 develop "expert" face processing capabilities? Several studies have highlighted how

532 important experience is in the processing of faces. For example, cataracts that

533 substantially decrease visual input from reaching the right hemisphere in infancy impair

534 "expert" face processing from completely developing (Le Grand, Mondloch, Maurer, \&

535 Brent, 2003). There is also evidence that perceptual narrowing and other complex aspects

536 of face processing continue to emerge over the first year of life, substantiating the 
537 hypothesis that experience is important in face processing (Kelley, Quinn, Slater, Lee,

538 Ge, Pascalis, 2008; Sai, 2005). However, other researchers have found that cortex

539 responds to faces extremely quickly after birth (Tzourio-Mazoyer et al., 2002), and

540 dispute the experience hypothesis (McKone, Crookes, Jeffery, \& Dilks, 2012). Our

541 results could suggest that other categories of stimuli with limited variability and increased

542 experience could tap into this rapid route.

543 Another theoretical framework that our results are relevant for is dual process

544 theory. Proponents of dual process theories have suggested that there are two processes

545 involved in cognition, the first an unconscious process (often thought of as procedural

546 learning), and the second a conscious, effortful process (i.e. explicit learning) (Barrett,

547 Tugade, \& Engle, 2004). In the categorization literature, others have proposed a dual

548 process model specific to categorization (COVIS), which has a procedural learning

549 component and a cognitively demanding, verbal hypothesis driven component, mediated

550 by the executive network (Ashby, Alfonso-Reese, Turken, \& Waldron, 1998; Maddox \&

551 Ing, 2005). It could be that faces access a rapid procedural mechanism while slower

552 categorization is dominated by the explicit process. However, other researchers have

553 criticised COVIS (Newell, Dunn, \& Kalish, 2011). From our current data is impossible to

554 determine whether our results are reflective of a dual process theory or are the result of a

555 single process that is more robust to faces. Future work should seek to examine this.

\section{Conclusions}

557 In conclusion, faces were detected with greater accuracy at fast reaction times than

558 houses, when they are distinct from the foil stimuli. Our data do not offer any support that 
559 these results are due to the contributions of the retinocollicular pathway, suggesting that a

560 traditional route to cortex is involved in the rapid detection of faces.

\section{Acknowledgements}

562 Jacob Matthews contributed helpful programming advice. The research was funded by a

563 Natural Sciences and Engineering Research Council of Canada (NSERC) Discovery

564 Grant (418293DG-2012). LC was supported by a Canadian Institute of Health Research

565 Master's scholarship.

566

567

568

569

570

571

572

573

574

575

576

577

578

579

580

581 
583 Ashby, F. G., Alfonso-Reese, L. A., Turken, A. U., \& Waldron, E. M. (1998). A

584 neuropsychological theory of multiple systems in category learning. Psychological

$585 \quad$ Review, 105(3), 442-81. Retrieved from

586 http://www.ncbi.nlm.nih.gov/pubmed/9697427

587 Awasthi, B., Sowman, P. F., Friedman, J., \& Williams, M. A. (2013). Distinct spatial

588 scale sensitivities for early categorization of faces and places : neuromagnetic and

589 behavioral findings. Frontiers in Human Neuroscience, 7(March), 1-11.

$590 \quad$ http://doi.org/10.3389/fnhum.2013.00091

591 Barragan-jason, G., Cauchoix, M., \& Barbeau, E. J. (2015). The neural speed of familiar 592 face recognition. Neuropsychologia, 75, 390-401.

593 http://doi.org/10.1016/j.neuropsychologia.2015.06.017

594 Barrett, L. F., Tugade, M. M., \& Engle, R. W. (2004). Individual Differences in Working 595 Memory Capacity and Dual-Process Theories of the Mind. Psychological Bulletin, 130(4), 553-573. http://doi.org/10.1037/0033-2909.130.4.553

597 Benevento, L. A., \& Standage, G. P. (1983). The organization of projections of the 598 retinorecipient and nonretinorecipient nuclei of the pretectal complex and layers of 599 the superior colliculus to the lateral pulvinar and medial pulvinar in the macaque 600 monkey. The Journal of Comparative Neurology, 217(3), 307-336.

601 http://doi.org/10.1002/cne.902170307

602 Bertini, C., Leo, F., \& Làdavas, E. (2008). Temporo-nasal asymmetry in multisensory 603 integration mediated by the Superior Colliculus. Brain Research, 1242(MAY), 37604 44. http://doi.org/10.1016/j.brainres.2008.03.087 
605 Burton, G. J., \& Moorhead, I. R. (1987). Color and spatial structure in natural scenes.

606 Applied Optics, 26(1), 157-170.

607 Cauchoix, S Crouzet, M. C. (2013). How plausible is a subcortical account of rapid visual 608 recognition? Frontiers in Human Neuroscience, 7(February), 1-4.

609 http://doi.org/10.3389/fnhum.2013.00039

610 Celebrini, S., Thorpe, S., Trotter, Y., \& Imbert, M. (1993). Dynamics of orientation

611 coding in area V1 of the awake primate. Visual Neuroscience, 10, 811-825.

612 Conley, M., Lachica, E. A., \& Casagrande, V. A. (1985). Demonstration of ipsilateral

613 retinocollicular projections in the tree shrew (Tupaia glis). Brain Research (Vol. $614346)$.

615 Crouzet, S. M., Kirchner, H., \& Thorpe, S. J. (2010). Fast saccades toward faces: face 616 detection in just 100 ms. Journal of Vision, 10(2010), 16.1-17.

$617 \quad$ http://doi.org/10.1167/10.4.16

618 Delorme, a., \& Thorpe, S. J. (2001). Face identification using one spike per neuron:

619 Resistance to image degradations. Neural Networks, 14(6-7), 795-803.

620 http://doi.org/10.1016/S0893-6080(01)00049-1

621 Fletcher-Watson, S., Findlay, J. M., Leekam, S. R., \& Benson, V. (2008). Rapid detection

622 of person information in a naturalistic scene. Perception, 37, 571-584.

623 http://doi.org/10.1068/p5705

624 Foundation, O. S. (2015). Estimating the reproducibility of psychological science.

$625 \quad$ Science, $349(6251)$. http://doi.org/10.1126/science.aac4716

626 Foxe, J. J., \& Simpson, G. V. (2002). Flow of activation from V1 to frontal cortex in

627 humans A framework for defining “ early " visual processing, 139-150. 
629 Gabay, S., Burlingham, C., \& Behrmann, M. (2014). The nature of face representations in 630 subcortical regions. Neuropsychologia, 59C, 35-46.

631 http://doi.org/10.1016/j.neuropsychologia.2014.04.010

632 Garvert, M. M., Friston, K. J., Dolan, R. J., \& Garrido, M. I. (2014). Subcortical

633 amygdala pathways enable rapid face processing. NeuroImage, 102, 309-316.

634 http://doi.org/10.1016/j.neuroimage.2014.07.047

635 Harrison, D. W. (2015). Brain Asymmetry and Neural Systems. Cham: Springer

636 International Publishing. http://doi.org/10.1007/978-3-319-13069-9

637 Haxby, J., Hoffman, E., \& Gobbini, M. (2000). The distributed human neural system for 638 face perception. Trends in Cognitive Sciences, 4(6), 223-233. Retrieved from 639 http://www.ncbi.nlm.nih.gov/pubmed/10827445

640 Johnson, M. H. (2005). Subcortical face processing. Nature Reviews. Neuroscience, $641 \quad 6(10), 766-74$. http://doi.org/10.1038/nrn1766

642 Jolij, J., \& Lamme, V. A. F. (2005). Repression of unconscious information by conscious 643 processing: Evidence from affective blindsight induced by transcranial magnetic $644 \quad$ stimulation. Proceedings of the National Academy of Sciences, 102(30), 10747$645 \quad$ 10751. http://doi.org/10.1073/pnas.0500834102

646 Jones, E. G., \& Burton, H. (1976). A projection from the medial pulvinar to the amygdala 647 in primates. Brain Research (Vol. 104).

648 Kanwisher, N., Mcdermott, J., \& Chun, M. M. (1997). The Fusiform Face Area : A 649 Module in Human Extrastriate Cortex Specialized for Face Perception. The Journal 650 of Neuroscience, 17(11), 4302-4311. 
651 Kanwisher, N., \& Yovel, G. (2006). The fusiform face area : a cortical region specialized 652 for the perception of faces, (November), 2109-2128.

653 http://doi.org/10.1098/rstb.2006.1934

654 Kelley, D, Quinn, P, Slater, A, Lee, K, Ge, L, Pascalis, O. (2008). The Other-Race Effect 655 Develops During Infancy, 18(12), 1084-1089. http://doi.org/10.1111/j.1467656 9280.2007.02029.x.The

657 Kirchner, H., \& Thorpe, S. J. (2006). Ultra-rapid object detection with saccadic eye 658 movements: Visual processing speed revisited. Vision Research, 46(11), 1762-1776. 659 http://doi.org/10.1016/j.visres.2005.10.002

660 Konig, P., Engel, A. K., Roelfsema, P. R., \& Singer, W. (n.d.). How Precise is Neuronal $661 \quad$ Synchronization?

662 Le Grand, R., Mondloch, C. J., Maurer, D., \& Brent, H. P. (2003). Expert face processing 663 requires visual input to the right hemisphere during infancy. Nature Neuroscience, 664 6(10), 1108-12. http://doi.org/10.1038/nn1121

665 Leopold, D. a, \& Rhodes, G. (2010). NIH Public Access, 124(3), 233-251.

666 http://doi.org/10.1037/a0019460.A

667 Liu, H., Agam, Y., Madsen, J. R., \& Kreiman, G. (2009). Article Timing, Timing, 668 Timing : Fast Decoding of Object Information from Intracranial Field Potentials in 669 Human Visual Cortex. Neuron, 62(2), 281-290.

670 http://doi.org/10.1016/j.neuron.2009.02.025

671 Liu, J., Harris, A., \& Kanwisher, N. (2002). Stages of processing in face perception: an 672 MEG study. Nature Neuroscience, 5(9), 910-916. http://doi.org/10.1038/nn909

673 Luo, Q., Holroyd, T., Majestic, C., Cheng, X., Schechter, J., \& Blair, R. J. (2010). 
674 Emotional Automaticity Is a Matter of Timing, 30(17), 5825-5829.

675 http://doi.org/10.1523/JNEUROSCI.BC-5668-09.2010

676 Maddox, T. W., \& Ing, D. A. (2005). Delayed Feedback Disrupts the Procedural-

677 Learning System but Not the Hypothesis-Testing System in Perceptual Category

678 Learning. Journal of Experimental Psychology, 21(1), 100-107.

679 http://doi.org/10.1037/0278-7393.31.1.100

680 Martin, C. B., McLean, D. A., O’Neil, E. B., \& Köhler, S. (2013). Distinct familiarity-

681 based response patterns for faces and buildings in perirhinal and parahippocampal

682 cortex. The Journal of Neuroscience: The Official Journal of the Society for

683 Neuroscience, 33(26), 10915-23. http://doi.org/10.1523/JNEUROSCI.0126-13.2013

684 McKone, E., Crookes, K., Jeffery, L., \& Dilks, D. D. (2012). A critical review of the

685 development of face recognition: experience is less important than previously

686 believed. Cognitive Neuropsychology, 29(1-2), 174-212.

687 http://doi.org/10.1080/02643294.2012.660138

688 Mende-Siedlecki, P., \& Verosky, S. (2013). Robust selectivity for faces in the human 689 amygdala in the absence of expressions, 2086-2106. http://doi.org/10.1162/jocn 690 Méndez-Bértolo, C., Moratti, S., Toledano, R., Lopez-Sosa, F., Martínez-Alvarez, R., 691 Mah, Y. H., ... Strange, B. A. (2016). A fast pathway for fear in human amygdala. $692 \quad$ Nature Neuroscience, 19(8), 1041-1049. http://doi.org/10.1038/nn.4324

693 Newell, B. R., Dunn, J. C., \& Kalish, M. (2011). Systems of Category Learning: Fact or 694 Fantasy? In The Psychology of Learning and Motivation (Vol. 54, pp. 167-215).

695 Academic Press. http://doi.org/10.1016/B978-0-12-385527-5.00006-1

696 Perry, V. H., \& Cowey, A. (1985). The ganglion cell and cone distributions in the 
monkey's retina: implications for central magnification factors. Vision Research, 25(12), 1795-810. Retrieved from http://www.ncbi.nlm.nih.gov/pubmed/3832605

699 Pitcher, D., Dilks, D. D., Saxe, R. R., Triantafyllou, C., \& Kanwisher, N. (2011).

700 Differential selectivity for dynamic versus static information in face-selective 701 cortical regions. NeuroImage, 56(4), 2356-2363.

702 http://doi.org/10.1016/j.neuroimage.2011.03.067

703 Pitcher, D., Walsh, V., Yovel, G., \& Aviv, T. (2007). TMS Evidence for the Involvement 704 of the Right Occipital Face Area in Early Face Processing. Current Biology, 17, $705 \quad$ 1568-1573. http://doi.org/10.1016/j.cub.2007.07.063

706 Pollack, J. G., \& Hickey, T. L. (1979). The distribution of retino-collicular axon terminals 707 in rhesus monkey. The Journal of Comparative Neurology, 185(4), 587-602.

$708 \quad$ http://doi.org/10.1002/cne.901850402

709 Rafal, R. D., Koller, K., Bultitude, J. H., Mullins, P., Ward, R., Mitchell, A. S., \& Bell,

710 A. H. (2015). Connectivity between the superior colliculus and the amygdala in

711 humans and macaque monkeys: virtual dissection with probabilistic DTI

712 tractography. Journal of Neurophysiology, 114(3), 1947-62.

713 http://doi.org/10.1152/jn.01016.2014

714 Sadeh, B., Podlipsky, I., Zhdanov, A., \& Yovel, G. (2010). Event-related potential and

715 functional MRI measures of face-selectivity are highly correlated: A simultaneous

716 ERP-fMRI investigation. Human Brain Mapping, 31(10), 1490-1501.

717 http://doi.org/10.1002/hbm.20952

718 Sai, F. Z. (2005). The Role of the Mother's Voice in Developing Mother's Face

719 Preference : Evidence for Intermodal Perception at Birth, 50, 29-50. 
720 http://doi.org/10.1002/icd.

721 Sato, W., Kochiyama, T., Uono, S., Matsuda, K., Usui, K., Inoue, Y., \& Toichi, M.

722 (2013). Rapid and multiple-stage activation of the human amygdala for processing 723 facial signals. Communicative \& Integrative Biology, 6(4), e24562.

724 http://doi.org/10.4161/cib.24562

725 Serre, T., Oliva, A., \& Poggio, T. (2007). A feedforward architecture accounts for rapid 726 categorization. Proceedings of the National Academy of Sciences, 104.

727 Sterling, P. (1973). Quantitative mapping with the electron microscope: retinal terminals 728 in the superior colliculus. Brain Research, 54, 347-354.

729 Stojanoski, Bobby, Cusack, R. (2016). Time to wave good-bye to phase scrambling :

730 Creating controlled scrambled images using diffeomorphic transformations, 731 14(2014), 1-16. http://doi.org/10.1167/14.12.6.doi

732 Sylvester, R., Josephs, O., Driver, J., \& Rees, G. (2007). Visual fMRI Responses in

733 Human Superior Colliculus Show a Temporal - Nasal Asymmetry That Is Absent in $734 \quad$ Lateral Geniculate and Visual Cortex, (Fries 1984), 1495-1502.

735 http://doi.org/10.1152/jn.00835.2006.

736 Tamietto, M., \& de Gelder, B. (2010). Neural bases of the non-conscious perception of

737 emotional signals. Nature Reviews. Neuroscience, 11(10), 697-709.

$738 \quad$ http://doi.org/10.1038/nrn2889

739 Tamietto, M., Pullens, P., De Gelder, B., Weiskrantz, L., \& Goebel, R. (2012).

740 Subcortical connections to human amygdala and changes following destruction of 741 the visual cortex. Current Biology, 22, 1449-1455.

$742 \quad$ http://doi.org/10.1016/j.cub.2012.06.006 
743 Tomalski, P., Johnson, M. H., \& Csibra, G. (2009). Temporal-nasal asymmetry of rapid

$744 \quad$ orienting to face-like stimuli. Neuroreport, 20(15), 1309-1312.

$745 \quad$ http://doi.org/10.1097/WNR.0b013e32832f0acd

746 Tong, F., Nakayama, K., Moscovitch, M., Weinrib, O., \& Kanwisher, N. (2000).

747 Response properties of the human fusiform face area. Cognitive Neuropsychology,

$748 \quad$ 17(1), 257-80. http://doi.org/10.1080/026432900380607

749 Tzourio-Mazoyer, N., De Schonen, S., Crivello, F., Reutter, B., Aujard, Y., \& Mazoyer,

750 B. (2002). Neural correlates of woman face processing by 2-month-old infants.

$751 \quad$ NeuroImage, 15(2), 454-61. http://doi.org/10.1006/nimg.2001.0979

752 VanRullen, R., Guyonneau, R., \& Thorpe, S. J. (2005). Spike times make sense. Trends

753 in Neurosciences, 28(1), 1-4. http://doi.org/10.1016/j.tins.2004.10.010

754 Vanrullen, R., \& Koch, C. (2001). Visual Selective Behavior Can Be Triggered by a

755 Feed-Forward Process. Journal of Cognitive Neuroscience, 15(2), 209-217.

756 Visconti di Oleggio Castello, M., \& Gobbini, M. I. (2015). Familiar Face Detection in

757180 ms. PloS One, 10(8), e0136548. http://doi.org/10.1371/journal.pone.0136548

758 Vuilleumier, P., Armony, J. L., Driver, J., \& Dolan, R. J. (2003). Distinct spatial

759 frequency sensitivities for processing faces and emotional expressions. Nature

$760 \quad$ Neuroscience, 6(6), 624-31. http://doi.org/10.1038/nn1057

761 Vuilleumier, P., Richardson, M. P., Armony, J. L., Driver, J., \& Dolan, R. J. (2004).

762 Distant influences of amygdala lesion on visual cortical activation during emotional

763 face processing, 7(11), 1271-1278. http://doi.org/10.1038/nn1341

764 